\title{
On the Spectrum
}

\section{of Holonomy Algebras}

\author{
Maria Cristina Abbati and Alessandro Manià \\ Dipartimento di Fisica, Università degli Studi di Milano and \\ Istituto Nazionale di Fisica Nucleare, Sezione di Milano, \\ via Celoria 16, 20133 Milano, Italy. \\ E-mail: "maria.cristina.abbati@mi.infn.it" \\ "alessandro.mania@mi.infn.it"
}

\begin{abstract}
Connections on a trivial bundle $M \times G$ can be identified with their holonomy maps, i.e. with homomorphisms of a groupoid of paths in $M$ into the gauge group $G$. For a connected compact $G$, various algebras depending on the set $\mathcal{A}$ of the smooth connections through their holonomy maps have been introduced in the literature, called cylindrical and holonomy algebras. We discuss the relations between these algebras and the consistence of their spectra.
\end{abstract}

Mathematical Subject Classification (2000): 81T13, 46J10.

Key words: holonomies, generalized connections, holonomy algebras.

\section{INTRODUCTION}

In the canonical treatment of Yang-Mills theories and, in general, of gauge theories, the space $\mathcal{A} / \operatorname{Gau}(P)$ represents the space of the physical configurations of the system. Here $\mathcal{A}$ denotes the set of the smooth connections on a principal bundle $P(M, G)$ and $\operatorname{Gau}(P)$ the group of gauge transformations, the base manifold $M$ is connected and $G$ is a connected compact Lie group.

A moment of paramount importance in developing the canonical quantization program for gauge theories invariant under diffeomorphisms, as proposed by Ashtekar et al. ([4], [5]), is to construct a compactification of the configuration space $\mathcal{A} / G a u(P)$. This compactification $\overline{\mathcal{A} / \operatorname{Gau}(P)}$, called quantum configuration space, is achieved 
by means of the immersion of $\mathcal{A} / \operatorname{Gau}(P)$ in the spectrum of a suitable $\mathbb{C}^{*}$-algebra of functions on $\mathcal{A} / \operatorname{Gau}(P)$, depending on holonomies on paths or on loops. To obtain the physical states for the corresponding quantum theory, a measure $\mu$ on the spectrum $\overline{\mathcal{A} / G a u(P)}$ is given and diffeomorphism invariant states are selected in the Hilbert space $L^{2}(\overline{\mathcal{A} / \operatorname{Gau}(P)}, \mu)$. This quantization procedure is known as the loop quantization and originates from the works of Rovelli and Smolin [25].

The starting point is the identification of a connection $A$ with its holonomy map $\gamma \mapsto H_{A}(\gamma)$, where $H_{A}(\gamma)$ means the parallel transport along the path $\gamma$; gauge equivalence classes of connections are identified with gauge equivalence classes of holonomy maps on loops ( [3], [20]).

Well known examples of gauge invariant functions depending on holonomies are the Wilson functions, defined - up to a scalar factor - as the maps $A \mapsto \operatorname{Tr} H_{A}(\lambda)$, where $\lambda$ is a loop. The generated $\mathbb{C}^{*}$ algebra is called holonomy algebra and depends on the differentiability class of loops. In the case that $G$ is $U(\mathrm{n})$ or $S U(\mathrm{n})$, the Wilson functions are separating on $\mathcal{A} / \operatorname{Gau}(P)$, so that $\mathcal{A} / \operatorname{Gau}(P)$ is densely immersed in the spectrum of the holonomy algebra.

For a general group $G$ the Wilson functions are no more separating, so one considers cylindrical functions, functions of the form $A \mapsto$ $f\left(H_{A}\left(\gamma_{1}\right), \ldots, H_{A}\left(\gamma_{n}\right)\right)$, for given paths $\gamma_{1}, \ldots, \gamma_{n}$. The $\mathbb{C}^{*}$-algebra generated by the cylindrical functions is called cylindrical algebra; the invariant cylindrical algebra is also defined. The cylindrical functions are separating on $\mathcal{A}$ so that $\mathcal{A}$ is densely embedded in the spectrum of the cylindrical algebra and, as well, $\mathcal{A} / \operatorname{Gau}(P)$ is densely embedded in the spectrum of the invariant cylindrical algebra.

The case of piecewise analytic loops and paths was the first to be investigated and many and nice results were obtained (see [6], [7], [8], 9], 23]). It was proved that the spectrum $\overline{\mathcal{A}}$ of the cylindrical algebra agrees with the space $\operatorname{Hom}(\operatorname{Path}(M), G)$ of the generalized connections, i.e. homomorphisms into $G$ of the groupoid $\operatorname{Path}(M)$ of piecewise analytic paths. The spectrum $\overline{\mathcal{A} / \operatorname{Gau}(P)}$ of the invariant analytical cylindrical algebra was proved to agree with the space $\operatorname{Hom}\left(\operatorname{Loop}_{\star}(M), G\right) / A d G$ of the $A d G$-equivalence classes of homomorphisms into $G$ of the group of piecewise analytic loops. Measures invariant under analytic diffeomorphisms have been constructed on this space by projective techniques using families of measures labelled by embedded graphs. For the so called natural measure a complete orthonormal set of states - the spin network states, depending on embedded graphs - was constructed and the invariance under analytic diffeomorphisms implemented. 
However the analytic setting is not satisfactory from the physical point of view, since invariance with respect to smooth diffeomorphisms is needed to use this quantization scheme for gravity.

In 110 the case of piecewise smoothly immersed paths was approached and the webs were introduced, special families of paths which play in the smooth immersive setting an analogous role to that of the embedded graphs in the analytic setting. Like every finite family of piecewise analytic paths depends on an embedded graph, i.e. every path can be written as a composition of edges belonging to the graph or of their inverses, so in the smooth immersive setting every finite family of paths depends on a web. This implies that the smooth immersive cylindrical algebra $C y l(\mathcal{A})$ is the limit of the algebras $C y l_{W}(\mathcal{A})$ generated by the cylindrical functions depending on a web $W$; dually, the spectrum of the cylindrical algebra is the projective limit of the spectra of $C y l_{W}(\mathcal{A})$. In [11 a diffeomorphism invariant measure was constructed using projective techniques, spin network states and spin web states were defined. The theory is quite involved, due to the fact that webs have a more involved behaviour than graphs and that the spectrum of the cylindrical algebra is not so simple to be characterized.

In this paper we introduce the cylindrical algebras, the invariant cylindrical algebras and the Wilson algebras in a general setting and illustrate their relations. These are algebras of functions defined on a subset $\mathcal{A}$ of $\operatorname{Hom}(\Lambda, G)$, the space of homomorphisms of a general groupoid $\Lambda$ into a connected compact Lie group $G$. We prove that the spectrum of the cylindrical algebras is always the closure $\overline{\mathcal{A}}$ of $\mathcal{A}$ in $\operatorname{Hom}(\Lambda, G)$.

For applications to gauge theories $\Lambda$ is a suitable groupoid of paths. The problem arises when $\overline{\mathcal{A}}=\operatorname{Hom}(\Lambda, G)$, where $\mathcal{A}$ is the set of connections. In the smooth immersive case this is true for a connected compact semisimple Lie group $G$, as proved in [22], but it is not true in the non semisimple case 19$]$. We characterize $\overline{\mathcal{A}}$ as a subset of $\operatorname{Hom}(\Lambda, G)$ for a general connected compact Lie group $G$, where $\Lambda$ is the groupoid Path $(M)$ of piecewise smooth immersed paths.

The paper is organized as follows. In section 2 we introduce the cylindrical algebras and we formulate in various forms the Approximation Condition, i.e. the condition that $\mathcal{A}$ is dense in $\operatorname{Hom}(\Lambda, G)$. We consider also the cylindrical algebras invariant under the action of $G^{M}$, where $M$ is the space of units of $\Lambda$ and we discuss their spectrum. In this section we develop and generalize some ideas proposed in $[12$.

In section 3 we define the Wilson functions on a subset $\mathcal{A}$ of $\operatorname{Hom}(\Lambda, G)$, where $\Lambda$ is a group. We investigate the spectrum of the generated $\mathbb{C}^{*}$ algebra and its relations with the invariant cylindrical algebras. 
In section 4 we apply the previous results to the holonomy algebras and to the cylindrical algebras on the space $\mathcal{A}$ of connections on the trivial bundle $M \times G$. The groupoid is now $\operatorname{Path}(M)$ or $\operatorname{Path}(M)$. Also the group Loop $(M)$ of piecewise analytic loops or the group $\operatorname{Loop}_{\star}(M)$ of piecewise smoothly immersed loops are considered. We discuss the relationship between the corresponding cylindrical algebras and the consistence of their spectrum.

In the last section we discuss cylindrical algebras in the general setting of non trivial bundles.

\section{Algebras of CylindRicAl FUnCtions}

Let we start recalling some well known results on Abelian $\mathbb{C}^{*}$-algebras of functions. Let $X$ be a non empty set, $B(X)$ the Abelian $\mathbb{C}^{*}$-algebra of bounded functions on $X$ and let $\mathcal{F} \subset B(X)$ a $\mathbb{C}^{*}$-subalgebra separating on $X$. The evaluation map associates to every $x \in X$ the multiplicative functional $e v_{x}: \mathcal{F} \rightarrow \mathbf{C}$, $e v_{x}(f)=f(x)$. By the assumption that $\mathcal{F}$ is separating the map ev $: x \mapsto e v_{x}$ is an embedding of $X$ in $\operatorname{Spec}(\mathcal{F})$. Moreover $X$ is dense in $\operatorname{Spec}(\mathcal{F})$ by the normality axiom. Therefore the following proposition holds.

Proposition 1. Let $\mathcal{F}$ be any $\mathbb{C}^{*}$-subalgebra of $B(X)$, separating on $X$. Then $X$ is injectively and densely embedded in $\operatorname{Spec}(\mathcal{F})$ by the evaluation map.

Let $X$ be a compact (Hausdorff) space. If $\mathcal{F}$ is the algebra $C(X)$ of the continuous functions, the evaluation map is an homeomorphism of $X$ onto $\operatorname{Spec}(\mathcal{F})$.

For every nonempty subset $Y$ of $X$, we associate the ${ }^{*}$-algebraic homomorphism $R_{Y}: C(X) \rightarrow C(Y) \cap B(Y), R_{Y}(F)=F_{Y}$ where $F_{Y}$ is the restriction of $F$ to $Y$. When $Y$ is closed, $R_{Y}$ is onto $C(Y)$ by Tietze extension Theorem; for $Y \subset Z \subset X$ and $Z$ closed, the range of $R_{Y}$ agrees with the set of restrictions to $Y$ of continuous functions on $Z$. We denote by $\mathcal{F}(Y)$ the $\mathbb{C}^{*}$-algebra generated by the range of $R_{Y}$. When $\bar{Y}=X$, the map $R_{Y}$ is an isometric isomorphism of $C(X)$ onto $\mathcal{F}(Y)$ whose inverse is the map which extends each $F \in \mathcal{F}(Y)$ to a continuous function on $X$. So we have that, for every $Y \subset X$, the $\mathbb{C}^{*}$-algebra $\mathcal{F}(Y)$ is naturally isomorphic to $C(\bar{Y})$.

Lemma 1. For every $Y \subset X$, the map $E_{Y}: \operatorname{Spec}(\mathcal{F}(Y)) \rightarrow X, E_{Y}(\varphi)=$ $x_{\varphi}$, with $x_{\varphi}$ given by

$$
F\left(x_{\varphi}\right)=\varphi\left(F_{Y}\right) \quad \forall F \in \mathcal{S} \subset C(X)
$$


for $\mathcal{S}$ separating on $X$, does not depend on $\mathcal{S}$ and is a continuous embedding and a homeomorphism onto $\bar{Y}$.

Proof. The map $E_{Y}$ is the composition of $R_{Y}^{\dagger}: \operatorname{Spec}(\mathcal{F}(Y)) \rightarrow$ $\operatorname{Spec}(C(X))$ with the inverse of the evaluation map ev $: X \rightarrow \operatorname{Spec}(C(X))$. The map $R_{Y}^{\dagger}$ is injective, since the image of $R_{Y}$ is dense in $\mathcal{F}(Y)$ and the evaluation map is a homeomorphism, since $X$ is compact. To prove continuity, recall that the topology on $X$ agrees with the $w^{*}$-topology. Let $\bar{x}=E_{Y}(\bar{\varphi})$ for a given $\bar{\varphi} \in \operatorname{Spec}(\mathcal{F}(Y))$; consider a finite family $\left\{F_{k}\right\}_{k=1, \ldots, r}$ in $C(X)$ and the open neighborhood of $\bar{x}$

$$
\left\{x \in X|| F_{k}(x)-F_{k}(\bar{x}) \mid<\epsilon \quad k=1, \ldots, r\right\} .
$$

Its inverse image by $E_{Y}$ is the set

$$
\left\{\varphi \in \operatorname{Spec}(\mathcal{F}(Y))|| \varphi\left(R_{Y} F_{k}\right)-\bar{\varphi}\left(R_{Y} F_{k}\right) \mid<\epsilon \quad k=1, \ldots, r\right\},
$$

an open set in the $w^{*}$-topology. So $E_{Y}$ is continuous, its image is closed and contains $\bar{Y}$, since $E_{Y}\left(e v_{y}\right)=y$ for every $y \in Y$.

By Proposition 1, every $\varphi \in \operatorname{Spec}(\mathcal{F}(Y))$ is the weak limit of some net of pure states $\left\{\varphi_{\mu}\right\}$ with $\varphi_{\mu}=e v_{y_{\mu}}, y_{\mu} \in Y$, hence $E_{Y}(\varphi)=\lim _{\mu} y_{\mu}$, proving that $E_{Y}$ is onto $\bar{Y}$.

Finally, we recall that every continuous injection of a compact space into a Hausdorff topological space is a homeomorphism with its image.

The condition that $Y$ is dense in $X$ will be called Approximation Condition on $Y$ and is conveniently stated in the following way:

For every $x \in X$, every finite family $\left\{F_{k}\right\}_{k=1, \ldots, r}$ in $C(X)$ and $\epsilon>0$ there exists $y \in Y$ such that

$$
\left|F_{k}(x)-F_{k}(y)\right|<\epsilon \quad k=1, \ldots, r .
$$

The condition can be restated by choosing $\left\{F_{k}\right\}_{k=1, \ldots, r} \subset \mathcal{S}$ if $\mathcal{S}$ is any separating subset of $C(X)$.

Now we assume that a compact group $G$ acts continuously on $X$. The quotient space $X / \mathrm{G}$ is a (Hausdorff) compact space and the canonical projection [ ] : $X \rightarrow X / \mathrm{G}$ is continuous, open and closed. The $\mathbb{C}^{*}$ algebra $C_{\mathrm{G}}(X)$ of the $\mathrm{G}$-invariant continuous functions on $X$ is identified with $C(X / \mathrm{G})$ by pull-back with the projection. As $X / \mathrm{G}$ is compact, $C_{\mathrm{G}}(X)$ is separating on $X / \mathrm{G}$.

For every G-invariant subset $Y$ of $X$ we get by simple topological arguments that $\bar{Y}$ is G-invariant, that $\overline{Y / G}=\bar{Y} / \mathrm{G}$ and that the map $R_{Y}$ is equivariant. We denote by $\mathcal{F}_{\mathrm{G}}(Y)$ the $\mathbb{C}^{*}$-subalgebra of $\mathrm{G}$-invariant functions in $\mathcal{F}(Y)$. 
Lemma 2. For every $\mathrm{G}$-invariant $Y \subset X$, the $\operatorname{map} I_{Y}: \operatorname{Spec}\left(\mathcal{F}_{\mathrm{G}}(Y)\right) \rightarrow$ $X / \mathrm{G}, I_{Y}(\varphi)=\left[x_{\varphi}\right]$, where $x_{\varphi} \in X$ satisfies

$$
F\left(x_{\varphi}\right)=\varphi\left(R_{Y} F\right) \quad \forall F \in \mathcal{S} \subset C_{\mathrm{G}}(X)
$$

for $\mathcal{S}$ separating on $X / G$, does not depend on $\mathcal{S}$ and is a continuous embedding and a homeomorphism onto $\overline{Y / G}$. $I_{Y}$ is a homeomorphism onto $X / \mathrm{G}$ if and only if $Y$ satisfies the Approximation Condition.

Proof. We identify $C_{\mathrm{G}}(X)$ with $C(X / \mathrm{G})$ and $\mathcal{F}_{\mathrm{G}}(Y)$ with $\mathcal{F}(Y / \mathrm{G})$; then we can apply Lemma 1 to $\mathcal{F}(Y / G)$.

For the last statement, we remark that $\overline{Y / \mathrm{G}}=\bar{Y} / \mathrm{G}$ and equals $X / \mathrm{G}$ if and only if $\bar{Y}=X$. This follows easily by the G-invariance of $\bar{Y}$.

As an immediate consequence of the previous arguments we obtain the canonical isomorphisms

$$
\mathcal{F}_{\mathrm{G}}(Y) \equiv C_{\mathrm{G}}(\bar{Y}) \equiv C(\bar{Y} / \mathrm{G}) .
$$

When $Y$ is G-invariant the Approximation Condition on $Y / \mathrm{G}$ can be stated on $Y / \mathrm{G}$ in terms of $\mathrm{G}$-invariant functions, as follows:

For every $x \in X$, every finite family $\left\{F_{k}\right\}_{k=1, \ldots, r} \subset C_{\mathrm{G}}(X)$ and $\epsilon>0$ there exists $y \in Y$ such that

$$
\left.\mid F_{k}(x)\right)-F_{k}(y) \mid<\epsilon \quad k=1, \ldots, r .
$$

The condition can be restated by choosing the functions $F_{k}$ in any separating subset $\mathcal{S} \subset C_{\mathrm{G}}(X)$.

More generally, let $\bar{Y}$ be G-invariant but $Y$ be not necessarily Ginvariant. This can be true, e.g., if the Approximation Condition on $Y$ holds. Using the isomorphism $\mathcal{F}(Y) \equiv C(\bar{Y})$, we can again consider the subalgebra $\mathcal{F}_{\mathrm{G}}(Y)$ of the functions $F \in \mathcal{F}(Y)$ whose extension is G-invariant and we have

$$
\operatorname{Spec}\left(\mathcal{F}_{\mathrm{G}}(Y)\right) \equiv \bar{Y} / \mathrm{G} .
$$

Now we come to the cylindrical algebras. Let us begin with the definition of groupoid.

A groupoid is a set $\Lambda$ endowed with a binary composition law satisfying:

i) to every $\lambda \in \Lambda$ an element $\lambda^{-1}$ (the inverse) is associated such that $r(\lambda)=\lambda \lambda^{-1}$ and $s(\lambda)=\lambda^{-1} \lambda$ exist and are the right and the left unit of $\lambda$, respectively;

ii) for $\lambda, \eta \in \Lambda$ the product $\lambda \eta$ exists if and only if $r(\eta)=s(\lambda)$;

iii) when defined, the product is associative. 
We denote by $M(\Lambda)$ the set of units of $\Lambda$, i.e. the elements of $\Lambda$ of the form $\lambda \lambda^{-1}$ for some $\lambda \in \Lambda$. A groupoid $\Lambda$ is a group if and only if $M(\Lambda)$ is a singleton.

Let $G$ denote a closed subgroup of $U(\mathrm{n})$, the group of unitary matrices in dimension $\mathrm{n}$. The set of homomorphisms $H: \Lambda \rightarrow G$, denoted by $\operatorname{Hom}(\Lambda, G)$, is a compact space since it is closed in $G^{\Lambda}$, a compact group in the Tychonoff product topology.

For a continuous function $f: G^{m} \rightarrow \mathbb{C}$ and a finite family $\left\{\lambda_{k}\right\}_{k=1, \ldots, m}$ in $\Lambda$, the continuous function $F_{\lambda_{1}, \ldots, \lambda_{m} ; f}: \operatorname{Hom}(\Lambda, G) \rightarrow \mathbb{C}$ defined by

$$
F_{\lambda_{1}, \ldots, \lambda_{m} ; f}(H)=f\left(H\left(\lambda_{1}\right), H\left(\lambda_{2}\right), \ldots, H\left(\lambda_{m}\right)\right),
$$

is called cylindrical function. The cylindrical functions form a normed *-algebra $\operatorname{cyl}(\operatorname{Hom}(\Lambda, G))$, whose completion is a $\mathbb{C}^{*}$-algebra, denoted by $\operatorname{Cyl}(\operatorname{Hom}(\Lambda, G))$. For any subset $\mathcal{A}$ of $\operatorname{Hom}(\Lambda, G)$, the ${ }^{*}$-algebra of the restrictions to $\mathcal{A}$ of the cylindrical functions is denoted by $\operatorname{cyl}(\mathcal{A})$ and the completion of $\operatorname{cyl}(\mathcal{A})$ by $\operatorname{Cyl}(\mathcal{A})$.

For $i, j=1, \ldots, \mathrm{n}$ and $\lambda \in \Lambda$, the cylindrical function $\Phi_{i, j ; \lambda}$ defined by

$$
\Phi_{i, j ; \lambda}(H)=H(\lambda)_{i, j} \quad H \in H o m(\Lambda, G),
$$

where $H(\lambda)_{i, j}$ denotes the corresponding matrix elements of $H(\lambda)$, will be called representative function. The representative functions are separating on $\operatorname{Hom}(\Lambda, G)$, hence their restrictions are separating on every subset $\mathcal{A}$. By Proposition 1 the evaluation map is a dense embedding of $\mathcal{A}$ in $\operatorname{Spec}(\operatorname{Cyl}(\mathcal{A}))$.

When $\mathcal{A}$ is closed, the Weierstrass Theorem gives $C y l(\mathcal{A})=C(\mathcal{A})$; as a special case we have $C y l(H o m(\Lambda, G))=C(\operatorname{Hom}(\Lambda, G))$. Recalling that $\mathcal{F}(\mathcal{A})$ is the $\mathbb{C}^{*}$-algebra generated by the range of the restriction map $R_{\mathcal{A}}: C(\operatorname{Hom}(\Lambda, G)) \rightarrow C(\mathcal{A})$, we get easily that $\mathcal{F}(\mathcal{A})=C y l(\mathcal{A})$. As a consequence of Lemma 1 we obtain the following theorem.

Theorem 1. For every $\mathcal{A} \subset \operatorname{Hom}(\Lambda, G)$, the map $E_{\mathcal{A}}: \operatorname{Spec}(C y l(\mathcal{A})) \rightarrow$ $\operatorname{Hom}(\Lambda, G), E_{\mathcal{A}}(\varphi)=H_{\varphi}$, with $H_{\varphi}: \Lambda \rightarrow G$ given by

$$
\Phi_{i, j ; \lambda}\left(H_{\varphi}\right)=\varphi\left(\Phi_{i, j ; \lambda}\right)
$$

for every representative function $\Phi_{i, j ; \lambda}$, is a continuous embedding and a homeomorphism onto $\overline{\mathcal{A}}$. The map $E_{\mathcal{A}}$ is onto $\operatorname{Hom}(\Lambda, G)$ if and only if the Approximation Condition on $\mathcal{A}$ is satisfied.

In this setting, the Approximation Condition on $\mathcal{A}$ is conveniently stated in the following way: 
For every $H$ in $\operatorname{Hom}(\Lambda, G)$, every finite family $\left\{\lambda_{k}\right\}_{k=1, \ldots, r} \subset \Lambda$ and $\epsilon>0$, there exists $H_{A} \in \mathcal{A}$ such that

$$
\left\|H\left(\lambda_{k}\right)-H_{A}\left(\lambda_{k}\right)\right\|<\epsilon \quad k=1, \ldots, r .
$$

The condition can be also restated as follows:

For every $H$ in $\operatorname{Hom}(\Lambda, G)$, every finite system of representative functions $\left\{\Phi_{i_{k}, j_{k} ; \lambda_{k}}\right\}_{k=1, \ldots, r}$ and $\epsilon>0$, there exists $H_{A} \in \mathcal{A}$ such that

$$
\left|\Phi_{i_{k}, j_{k} ; \lambda_{k}}(H)-\Phi_{i_{k}, j_{k} ; \lambda_{k}}\left(H_{A}\right)\right|<\epsilon \quad k=1, \ldots, r
$$

The Tychonoff and the $w^{*}$-topology agree on $\operatorname{Hom}(\Lambda, G)$. In the first version of the Approximation Condition, the density of $\mathcal{A}$ in $\operatorname{Hom}(\Lambda, G)$ is expressed in terms of the usual basis for the Tychonoff topology, in the second one using a basis for the $w^{*}$-topology.

From now on, we denote $M(\Lambda)$ shortly by $M$ and consider the natural continuous right action of the compact group $G^{M}$ on $\operatorname{Hom}(\Lambda, G)$ given by

$$
(H . g)(\lambda)=g^{-1}(r(\lambda)) H(\lambda) g(s(\lambda))
$$

The dual isometric action on $C(\operatorname{Hom}(\Lambda, G))$ is defined by $g F(H)=$ $F\left(H . g^{-1}\right)$ for every $H \in H o m(\Lambda, G)$ and $g \in G^{M}$. For a $G^{M}$-invariant $\mathcal{A} \subset \operatorname{Hom}(\Lambda, G)$, we denote by $C y l_{G^{M}}(\mathcal{A})$ the $\mathbb{C}^{*}$-subalgebra of the $G^{M}$-invariant functions of $\operatorname{Cyl}(\mathcal{A})$.

By Lemma 2 we obtain the following theorem.

Theorem 2. Let $\mathcal{A}$ be a $G^{M}$-invariant subset of $\operatorname{Hom}(\Lambda, G)$. The map $I_{\mathcal{A}}: \operatorname{Spec}\left(\operatorname{Cyl}_{G^{M}}(\mathcal{A})\right) \rightarrow \operatorname{Hom}(\Lambda, G) / G^{M}, I_{\mathcal{A}}(\varphi)=\left[H_{\varphi}\right]$, where $H_{\varphi}$ satisfies

$$
F\left(H_{\varphi}\right)=\varphi\left(R_{\mathcal{A}} F\right) \quad \forall F \in \mathcal{S} \subset C \operatorname{Cyl}_{G^{M}}(\operatorname{Hom}(\Lambda, G))
$$

for $\mathcal{S}$ separating on $\left(\operatorname{Hom}(\Lambda, G) / G^{M}\right.$, does not depend on $\mathcal{S}$ and is a continuous embedding and a homeomorphism onto $\overline{\mathcal{A}} / G^{M}$. The map $I_{\mathcal{A}}$ is onto $\operatorname{Hom}(\Lambda, G) / G^{M}$ if and only if the Approximation Condition on $\mathcal{A}$ is satisfied.

In the special case that $\Lambda$ is a group, the set of units is just $\{e\}$, so $G^{M}=G$ and its action on $\operatorname{Hom}(\Lambda, G)$ is given by $(H . a)(\lambda)=$ $a^{-1} H(\lambda) a=A d_{a^{-1}} H(\lambda)$ for $a \in G$. For any $A d G$-invariant $\mathcal{A}$, we have

$$
\operatorname{Spec}\left(C y l_{A d G}(\mathcal{A})\right)=\operatorname{Spec}(C y l(\mathcal{A})) / A d G=\overline{\mathcal{A}} / A d G .
$$


A natural choice for $\mathcal{S}$ in Theorem 2 is $\operatorname{cyl}_{G^{M}}(\operatorname{Hom}(\Lambda, G))$, the *subalgebra of the $G^{M}$-invariant cylindrical functions. Let us show that $\operatorname{cyl}_{G^{M}}(\operatorname{Hom}(\Lambda, G))$ is separating on $\operatorname{Hom}(\Lambda, G) / G^{M}$.

Theorem 3. Let $d g$ denote the normalized Haar measure on $G^{M}$.

i) The mean value map \langle\rangle$: C(\operatorname{Hom}(\Lambda, G)) \rightarrow C_{G^{M}}(\operatorname{Hom}(\Lambda, G))$ defined by

$$
\langle F\rangle=\int_{G^{M}} g F d g
$$

restricts to a continuous surjection from $\operatorname{cyl}(\operatorname{Hom}(\Lambda, G))$ onto the *subalgebra $\operatorname{cyl}_{G^{M}}(H o m(\Lambda, G))$ of $G^{M}$-invariant cylindrical functions.

ii) The *-algebra cyl $G_{G^{M}}(\operatorname{Hom}(\Lambda, G))$ is separating on $\operatorname{Hom}(\Lambda, G) / G^{M}$.

Proof. i) The only non trivial point in the first statement is that the mean value map sends $\operatorname{cyl}(\operatorname{Hom}(\Lambda, G))$ into $\operatorname{cyl}_{G^{M}}(\operatorname{Hom}(\Lambda, G))$. Actually, for every cylindrical function $F=F_{\lambda_{1}, \ldots, \lambda_{m} ; f}$ we have $(g F)(H)=f\left(g\left(r\left(\lambda_{1}\right)\right) H\left(\lambda_{1}\right) g^{-1}\left(s\left(\lambda_{1}\right)\right), \ldots, g\left(r\left(\lambda_{m}\right)\right) H\left(\lambda_{m}\right) g^{-1}\left(s\left(\lambda_{m}\right)\right)\right)$. The elements of $G^{2 m}$ of the form $\left(g\left(r\left(\lambda_{1}\right)\right), g\left(s\left(\lambda_{1}\right)\right), \ldots, g\left(r\left(\lambda_{m}\right)\right), g\left(s\left(\lambda_{m}\right)\right)\right)$ for some $g \in G^{M}$ form a subgroup $\Gamma$ isomorphic to $G^{d}$, where $d$ denotes the number of distinct units in $M$ which are of the form $r\left(\lambda_{i}\right)$ or $s\left(\lambda_{i}\right)$ for some $i=1, . ., m$. Therefore

$$
\begin{gathered}
\langle F\rangle(H)=\int_{G^{M}} f\left(g\left(r\left(\lambda_{1}\right)\right) H\left(\lambda_{1}\right) g^{-1}\left(s\left(\lambda_{1}\right)\right), \ldots, g\left(r\left(\lambda_{m}\right)\right) H\left(\lambda_{m}\right) g^{-1}\left(s\left(\lambda_{m}\right)\right)\right) d g \\
=\int_{\Gamma}\left(a^{-1} f\right)\left(H\left(\lambda_{1}\right), \ldots ., H\left(\lambda_{m}\right)\right) d a
\end{gathered}
$$

where for $a \in G^{2 m}, a^{-1} f$ is given by $a^{-1} f\left(g_{1}, \ldots, g_{m}\right)=f\left(a_{1} g_{1} a_{2}^{-1}, \ldots, a_{2 m-1} g_{m} a_{2 m}\right)$ and $d a$ denotes the Haar measure on $\Gamma \equiv G^{d}$. Thus $\langle F\rangle$ is a cylindrical function, since $\langle F\rangle=F_{\lambda_{1}, \ldots, \lambda_{m} ; \tilde{f}}$ where $\tilde{f}$, given by $\tilde{f}\left(g_{1}, \ldots, g_{m}\right)=$ $\int_{\Gamma}\left(a^{-1} f\right)\left(g_{1}, \ldots, g_{m}\right) d a$, is continuous.

ii) Let $F_{n} \in \operatorname{cyl}(\operatorname{Hom}(\Lambda, G))$ converge to $F \in C y l_{G^{M}}(\operatorname{Hom}(\Lambda, G))$. Then $\left\langle F_{n}\right\rangle \rightarrow\langle F\rangle=F$, proving that $\operatorname{cyl}_{G^{M}}(\operatorname{Hom}(\Lambda, G))$ is dense in $\operatorname{Cyl}_{G^{M}}(\operatorname{Hom}(\Lambda, G))$. This is equivalent to say that $\operatorname{cyl}_{G^{M}}(\operatorname{Hom}(\Lambda, G))$ is separating.

Fix a point $\star$ in $M$. The elements $\lambda$ of $\Lambda$ satisfying $s(\lambda)=r(\lambda)=\star$ form a group, denoted by $\Lambda_{\star}$. The restriction map defines a continuous projection

$$
\mathcal{P}_{\star}: \operatorname{Hom}(\Lambda, G) \rightarrow \operatorname{Hom}\left(\Lambda_{\star}, G\right),
$$

which is equivariant w.r.t. the action of $G^{M}$; actually $(H g)_{\star}=A d_{g(\star)^{-1}} H_{\star}$, where $H_{\star}=\mathcal{P}_{\star}(H)$. 
We say that $M$ is $\Lambda$-connected if , for every $s, r \in M$, there exists $\lambda \in \Lambda$ with $s=s(\lambda)$ and $r=r(\lambda)$. If $M$ is $\Lambda$-connected, $\mathcal{P}_{\star}$ is onto and quotients to a homeomorphism

$$
\mathcal{Q}_{\star}: \operatorname{Hom}(\Lambda, G) / G^{M} \rightarrow \operatorname{Hom}\left(\Lambda_{\star}, G\right) / A d G .
$$

This is proved following an argument of Velhinho in [27]. Let $G_{\star}^{M}$ the compact subgroup of maps $g \in G^{M}$ such that $g(\star)=1$. For every $x \in M$, we fix a unique $e_{x} \in \Lambda$ with $s\left(e_{x}\right)=\star$ and $r\left(e_{x}\right)=x$, choosing $e_{\star}=\star$. To every $H$ we associate $g_{H} \in G_{\star}^{M}$ by defining $g_{H}(x)=H\left(e_{x}\right)$. The map

$$
\Theta: \operatorname{Hom}(\Lambda, G) \rightarrow \operatorname{Hom}\left(\Lambda_{\star}, G\right) \times G_{\star}^{M}, \quad H \mapsto\left(H_{\star}, g_{H}\right)
$$

is a homeomorphism. Actually, $\mathcal{P}_{\star}$ is onto since for $h \in \operatorname{Hom}\left(\Lambda_{\star}, G\right)$ we define $H \in H o m(\Lambda, G)$ such that $H_{\star}=h$ by $H(\lambda)=h\left(e_{r(\lambda)}^{-1} \lambda e_{s(\lambda)}\right)$. The map $\Theta$ is continuous and it is onto since, for every $H \in \operatorname{Hom}(\Lambda, G)$ and $g \in G_{\star}^{M}$, we have $\Theta\left(H .\left(g_{H} g^{-1}\right)\right)=\left(H_{\star}, g\right)$. The inverse is defined by $\left(H_{\star}, g\right) \mapsto H^{\prime} .\left(g_{H^{\prime}} g^{-1}\right)$ for any $H^{\prime}$ such that $\mathcal{P}_{\star}\left(H^{\prime}\right)=H_{\star}$.

A continuous action of $G^{M}$ on $\operatorname{Hom}\left(\Lambda_{\star}, G\right) \times G_{\star}^{M}$ is given by

$$
\left(H_{\star}, g^{\prime}\right) \cdot g=\left(A d_{g^{-1}(\star)} H_{\star}, R_{g}\left(g^{\prime}\right)\right),
$$

where $R_{g}\left(g^{\prime}\right)(x)=g(x)^{-1} g^{\prime}(x) g(\star)$. It is clear that $\Theta$ quotients to the wanted homeomorphism $\mathcal{Q}_{\star}$.

If $\mathcal{A}$ is $G^{M}$-invariant, then $\mathcal{A}_{\star}=\mathcal{P}_{\star}(\mathcal{A})$ is $A d G$-invariant; the restriction of $\mathcal{Q}_{\star}$ is a homeomorphism of $\mathcal{A} / G^{M}$ onto $\mathcal{A}_{\star} / A d G$ and, obviously, of $\overline{\mathcal{A} / G^{M}}$ onto $\overline{\mathcal{A}_{\star} / A d G}$. By duality we obtain the isomorphism

$$
C y l_{G^{M}}(\mathcal{A}) \equiv C y l_{A d G}\left(\mathcal{A}_{\star}\right) \text {. }
$$

If the Approximation Condition on $\mathcal{A}$ is satisfied, we get

$$
\operatorname{Spec}\left(\operatorname{Cyl} l_{G^{M}}(\mathcal{A})\right) \equiv \operatorname{Hom}\left(\Lambda_{\star}, G\right) / A d G .
$$

We take now into account the case that $\overline{\mathcal{A}}$ is $G^{M}$-invariant but $\mathcal{A}$ is not necessarily $G^{M}$-invariant.

Theorem 4. If $\overline{\mathcal{A}}$ is $G^{M}$-invariant and $\mathcal{A}_{\star}$ is $A d G$-invariant, then

$$
\operatorname{Spec}\left(C y l_{G^{M}}(\mathcal{A})\right) \equiv \overline{\mathcal{A}} / G^{M} \equiv \overline{\mathcal{A}_{\star} / A d G} \equiv \operatorname{Spec}\left(C y l_{\text {AdG }}\left(\mathcal{A}_{\star}\right)\right)
$$

where $\operatorname{Cyl}_{G^{M}}(\mathcal{A})$ denotes the $\mathbb{C}^{*}$-algebra of restrictions to $\mathcal{A}$ of functions in $\operatorname{Cyl}_{G^{M}}(\overline{\mathcal{A}})$. Moreover $\overline{\mathcal{A}}_{\star}=\operatorname{Hom}\left(\Lambda_{\star}, G\right)$ if and only if $\overline{\mathcal{A}}=$ $\operatorname{Hom}(\Lambda, G)$.

Proof. By definition, $C y l_{G^{M}}(\mathcal{A})$ is isomorphic to $C y l_{G^{M}}(\overline{\mathcal{A}})$ whose spectrum is $\overline{\mathcal{A}} / G^{M}$, since $\overline{\mathcal{A}}$ is $G^{M}$-invariant. The restriction of $\mathcal{Q}_{\star}$ to $\overline{\mathcal{A}} / G^{M}$ is a homeomorphism onto $(\overline{\mathcal{A}})_{\star} / A d G$. By continuity of $\mathcal{P}_{\star}$, 
the set $(\overline{\mathcal{A}})_{\star}$ is closed, hence the relation $\mathcal{A}_{\star} \subset(\overline{\mathcal{A}})_{\star} \subset \overline{\mathcal{A}_{\star}}$ implies that $(\overline{\mathcal{A}})_{\star}=\overline{\mathcal{A}_{\star}}$. Moreover the $A d G$-invariance of $\mathcal{A}_{\star}$ gives $\overline{\mathcal{A}_{\star}} / A d G=$ $\overline{\mathcal{A}_{\star} / A d G}$. Hence we have $\mathcal{Q}_{\star}\left(\overline{\mathcal{A}} / G^{M}\right)=\overline{\mathcal{A}_{\star} / A d G}$.

To prove the second statement, let $\overline{\mathcal{A}_{\star}}=\operatorname{Hom}\left(\Lambda_{\star}, G\right)$. This is equivalent to $\overline{\mathcal{A}}_{\star} / A d G=(\overline{\mathcal{A}})_{\star} / A d G=\operatorname{Hom}\left(\Lambda_{\star}, G\right) / A d G$. By the homeomorphism $\mathcal{Q}_{\star}$ we obtain $\overline{\mathcal{A}} / G^{M}=\operatorname{Hom}(\Lambda, G) / G^{M}$. The $G^{M_{-}}$ invariance of $\overline{\mathcal{A}}$ gives $\overline{\mathcal{A}}=\operatorname{Hom}(\Lambda, G)$.

\section{The Wilson Algebras}

In this section $\Lambda$ will be a group and $G$ a closed subgroup of $U(\mathbf{n})$. To every $\lambda \in \Lambda$ we associate the cylindrical map $T_{\lambda}$ on $\operatorname{Hom}(\Lambda, G)$ defined by

$$
T_{\lambda}(H)=\frac{1}{\mathrm{n}} \operatorname{Tr}(H(\lambda)) .
$$

These functions are called Wilson functions. To every $\mathcal{A} \subset \operatorname{Hom}(\Lambda, G)$ we associate the Wilson $\mathbb{C}^{*}$-algebra of $\mathcal{A}$, denoted by $\mathfrak{H}_{\mathcal{A}}$, which is the $\mathbb{C}^{*}$-algebra generated by the Wilson functions restricted to $\mathcal{A}$.

If $H, H^{\prime} \in H \operatorname{Hom}(\Lambda, G)$ are equivalent representations of $\Lambda$ we have $T_{\lambda}(H)=T_{\lambda}\left(H^{\prime}\right)$ for every $\lambda \in \Lambda$. Besides, it is well known that equivalent homomorphisms of any group in $U(\mathrm{n})$ are unitarily equivalent. So we consider the quotient of $\operatorname{Hom}(\Lambda, G)$ by unitary equivalence. For $H \in \operatorname{Hom}(\Lambda, G)$ we denote by $\widehat{H}$ its unitary equivalence class and by $\widehat{\mathcal{A}}$ the set of unitary equivalence classes of homomorphisms in $\mathcal{A}$.

The Wilson functions are separating on $\widehat{\operatorname{Hom}}(\Lambda, G)$, as follows from the next proposition.

Proposition 2. Let $\Lambda$ be a group, $H$ and $H^{\prime}$ in $H \operatorname{Hom}(\Lambda, G)$. If $T_{\lambda}(H)=$ $T_{\lambda}\left(H^{\prime}\right)$ for every $\lambda \in \Lambda$, then $H$ and $H^{\prime}$ are unitarily equivalent.

Proof. To begin with, let us consider a topological group $\Lambda$ and continuous homomorphisms. We recall that there exists a compact (Hausdorff) group $C p t(\Lambda)$, called the associated compact group of $\Lambda$, and a homomorphism $\varkappa: \Lambda \rightarrow \operatorname{Cpt}(\Lambda)$ with dense range such that the following universality property holds: to any $G$-valued continuous representation $H$ of $\Lambda$ one can associate a unique representation $K$ : $\operatorname{Cpt}(\Lambda) \rightarrow G$, such that $H=K \circ \varkappa$ (see e.g. [14]). By density of $\varkappa(\Lambda)$ in $C p t(\Lambda)$, the equality $T_{\lambda}(H)=T_{\lambda}\left(H^{\prime}\right)$ for every $\lambda \in \Lambda$ implies that $\operatorname{Tr}(K(\xi))=\operatorname{Tr}\left(K^{\prime}(\xi)\right)$, for every $\xi \in \operatorname{Cpt}(\Lambda)$. By a well known theorem on the representations of compact groups (see e.g. [13) $K$ and $K^{\prime}$ are equivalent. This implies that $H$ and $H^{\prime}$ are equivalent, hence unitarily equivalent. 
If no topology is assumed on $\Lambda$, we give $\Lambda$ the topology induced by all homomorphisms $H: \Lambda \rightarrow G$, so that $\Lambda$ becomes a topological group and $H$ and $H^{\prime}$ continuous representations. Therefore we are reduced to the previous case.

The Proposition 2 assures that $\mathfrak{H}_{\mathcal{A}}$ is separating on $\widehat{\mathcal{A}}$, hence $\widehat{\mathcal{A}}$ is densely embedded in $\operatorname{Spec}\left(\mathfrak{H}_{\mathcal{A}}\right)$. To characterize $\operatorname{Spec}\left(\mathfrak{H}_{\mathcal{A}}\right)$ it is convenient to consider $\mathcal{A}$ as a subset of $\operatorname{Hom}(\Lambda, U(\mathrm{n}))$ and identify $\widehat{\mathcal{A}}$ with the subset of $\operatorname{Hom}(\Lambda, U(\mathrm{n})) / A d U(\mathrm{n})$ obtained by applying to $\mathcal{A}$ the projection ^ $: \operatorname{Hom}(\Lambda, U(\mathrm{n})) \rightarrow \operatorname{Hom}(\Lambda, U(\mathrm{n})) / A d U(\mathrm{n})$. The Wilson functions are separating on $\operatorname{Hom}(\Lambda, U(\mathrm{n})) / A d U(\mathrm{n})$, so they generate the algebra of continuous functions on $\operatorname{Hom}(\Lambda, U(\mathrm{n})) / A d U(\mathrm{n})$. Then, by Lemma 目, $\operatorname{Spec}\left(\mathfrak{H}_{\mathcal{A}}\right)$ is homeomorphic to the closure of $\widehat{\mathcal{A}}$ in $\operatorname{Hom}(\Lambda, U(\mathrm{n})) / A d U(\mathrm{n})$.

Wilson functions become relevant in gauge theories when $\mathcal{A}$ is $A d G$ invariant and $\widehat{\mathcal{A}}=\mathcal{A} / A d G$. To obtain this identification we have to specialize the group $G$. One can assume that for every $a \in G$ there exist $\lambda \in \Lambda$ and $H \in \mathcal{A}$ such that $a=H(\lambda)$. This is always satisfied in the cases arising in gauge theories. Under this assumption the condition $\widehat{\mathcal{A}}=\mathcal{A} / A d G$ implies that $G$ is a normal subgroup of $U(\mathrm{n})$.

Conversely, if a group $G$ is a closed normal subgroup of $U(\mathrm{n})$, one gets $\widehat{\operatorname{Hom}}(\Lambda, G)=\operatorname{Hom}(\Lambda, G) / A d G$. This is a consequence of the following proposition.

Proposition 3. Let $G$ a closed normal subgroup $U(\mathrm{n})$. Then the conjugation classes w.r.t. $A d_{U(\mathrm{n})}$ and $A d_{G}$ coincide on $G$.

Proof. The smooth group homomorphism $\Phi: U(1) \times S U(\mathrm{n}) \rightarrow$ $U(\mathrm{n}), \quad \Phi(\alpha, S)=\alpha S$, is onto since, for $U \in U(\mathrm{n}), \Phi\left(\delta, \delta^{-1} U\right)=U$ for every $\delta \in U(1)$ such that $\delta^{\mathrm{n}}=\operatorname{det}(U)$. As $G$ is a closed normal subgroup of $U(\mathbf{n})$, then $\widetilde{G}=\Phi^{-1}(G)$ is a compact normal subgroup of $U(1) \times S U(\mathbf{n})$. Therefore $\pi_{s}(\widetilde{G})$ is a compact normal subgroup of $S U(\mathrm{n})$, where $\pi_{s}: U(1) \times S U(\mathrm{n}) \rightarrow S U(\mathrm{n})$ is the canonical projection. As $S U(\mathrm{n})$ is simple, compact and connected, we have two cases:

i) $\pi_{s}(\widetilde{G})=S U(\mathrm{n})$;

ii) $\pi_{s}(\widetilde{G})$ is a (finite) central subgroup of $S U(\mathbf{n})$.

We discuss separately these cases.

i) Let $U \in U(\mathbf{n})$ of the form $U=\alpha S$ with $\alpha \in U(1)$ and $S \in S U(\mathbf{n})$. By assumption i), there exists $(\beta, S) \in \widetilde{G}$, for some $\beta \in U(1)$, so that $h=\beta S$ belongs to $G$. For every $V \in U(\mathrm{n})$ we have $A d_{U} V=A d_{S} V=$ $A d_{h} V$. 
ii) If $\pi_{s}(\widetilde{G})$ is a central subgroup of $S U(\mathrm{n})$, then $\widetilde{G}$ is a central subgroup of $U(1) \times S U(\mathbf{n})$, so that $G$ is a central subgroup of $U(\mathbf{n})$. Thus the adjoint actions on $G$ are both trivial.

Then, let $G$ be a closed normal subgroup of $U(\mathrm{n})$. The Wilson functions become a separating set of cylindrical functions on $\operatorname{Hom}(\Lambda, G) / A d G=$ $\widehat{\operatorname{Hom}}(\Lambda, G)$, so they generate the algebra $C_{A d G}(\operatorname{Hom}(\Lambda, G))$. For every $A d G$-invariant subset $\mathcal{A}$ of $\operatorname{Hom}(\Lambda, G)$, we have $\widehat{\mathcal{A}}=\mathcal{A} / A d G$ and $\mathfrak{H}_{\mathcal{A}}=C y l_{A d G}(\mathcal{A})$.

The density of $\widehat{\mathcal{A}}$ in $\widehat{\operatorname{Hom}}(\Lambda, G)$ is assured by the Wilson Approximation Condition on $\mathcal{A}$, formulated as follows:

For every $H$ in $\operatorname{Hom}(\Lambda, G)$, every finite family $\left\{\lambda_{k}\right\}_{k=1, \ldots, r}$ of $\Lambda$ and $\epsilon>0$, there exists $H_{A} \in \mathcal{A}$ such that

$$
\left|\operatorname{Tr} H\left(\lambda_{k}\right)-\operatorname{Tr} H_{A}\left(\lambda_{k}\right)\right|<\epsilon \quad k=1, \ldots, r .
$$

Thus we have obtained the following theorem.

Theorem 5. Let $\Lambda$ be a group, $G$ a closed normal subgroup of $U(\mathbf{n})$ and $\mathcal{A}$ an $A d G$-invariant subset of $\operatorname{Hom}(\Lambda, G)$. Then:

i) $\mathfrak{H}_{\mathcal{A}}$ agrees with $C y l_{A d G}(\mathcal{A})$;

ii) the $\operatorname{map} I_{\mathcal{A}}: \operatorname{Spec}\left(\mathfrak{H}_{\mathcal{A}}\right) \rightarrow \widehat{\operatorname{Hom}}(\Lambda, G), I_{\mathcal{A}}(\varphi)=\left[H_{\varphi}\right]$, where $H_{\varphi}$ satisfies

$$
\varphi\left(T_{\lambda}\right)=\frac{1}{\mathrm{n}} \operatorname{Tr} H_{\varphi}(\lambda) \quad \lambda \in \Lambda,
$$

is a homeomorphism onto the closure of $\widehat{\mathcal{A}}$ in $\widehat{\operatorname{Hom}}(\Lambda, G)$;

iii) the embedding $I_{\mathcal{A}}$ is onto $\widehat{\operatorname{Hom}}(\Lambda, G)$ if and only if the Wilson Approximation Condition is satisfied.

\section{Application to Gauge Theories}

Now we discuss the applications to gauge theories of the statements proved in the above sections.

Let $M$ be a connected, orientable paracompact smooth manifold with $\operatorname{dim}(M)>1$. Then $M$ admits a compatible real analytic structure, which is unique up to diffeomorphisms.

We will start considering continuous piecewise smooth (or piecewise analytic) parametrized paths and loops $\gamma:[0,1] \rightarrow M$. Paths $\gamma$ and $\lambda$ with $\gamma(1)=\lambda(0)$ can be composed to get $\lambda \gamma:[0,1] \rightarrow M$ defined by

$$
(\lambda \gamma)(t)= \begin{cases}\gamma(2 t) & \text { if } 0 \leq t<1 / 2 \\ \lambda(2 t-1) & \text { if } 1 / 2 \leq t \leq 1\end{cases}
$$

The inverse $\gamma^{-1}$ is defined by $\gamma^{-1}(t)=\gamma(1-t)$. 
By immediate retracing we mean a parametrized piecewise smooth path $\gamma$ which factorizes as

$$
\gamma=\prod_{i}^{k}\left(\gamma_{i} \gamma_{i}^{-1}\right)
$$

Equivalence of piecewise smooth (or analytic) parametrized paths w.r.t. order preserving piecewise smooth (or piecewise analytic) reparametrizations and up to immediate retracings will be called elementary equivalence. A piecewise smooth (analytic) path is an elementary equivalence class of piecewise smooth (piecewise analytic) parametrized paths. For applications to gauge theories it is convenient to consider just paths which are piecewise smoothly immersed or constant. Paths can be composed and inverted, and form a groupoid, denoted by $\operatorname{Path}(M)$ in the piecewise analytic case and by $\operatorname{Path}(M)$ in the piecewise smooth immersive case. Obviously, one could also consider weaker differentiability conditions on paths and more general groupoids, as in [17] and [18.

Fix a base point $\star$ in $M$. A piecewise smooth (analytic) loop based on $\star$ is an elementary equivalence class of piecewise smooth (analytic) parametrized paths $\gamma$ with $\gamma(0)=\gamma(1)=\star$. Loops based on $\star$ form a group, denoted by Loop $(M)$ in the analytic case and by $\operatorname{Loop}_{\star}(M)$ in the smooth immersive case. The unit is the constant loop, also denoted by $\star$. If the base points are changed, one obtains isomorphic groups.

Let us consider now the trivial bundle $M \times G$, where $G$ is a connected closed subgroup of $U(\mathrm{n})$. For a smooth connection $A$ on $M \times G$, the holonomy map $H_{A}$ is defined, which associates to a path $\lambda$ the parallel transport along $\lambda$, identified with an element $H_{A}(\lambda)$ of $G$. We can identify the set $\mathcal{A}$ of smooth connections with the set of their associated holonomy maps on $\operatorname{Path}(M)$, on $\operatorname{Path}(M)$ [20] or even on more general groupoids.

Connections induce another equivalence relation on parametrized paths (of any class), called holonomy equivalence, where parametrized paths $\lambda$ and $\lambda^{\prime}$ are holonomy equivalent if

$$
\lambda^{\prime}(0)=\lambda(0), \lambda^{\prime}(1)=\lambda(1) \quad \text { and } \quad H_{A}\left(\lambda^{\prime} \lambda^{-1}\right)=e \quad \forall A \in \mathcal{A} .
$$

The holonomy equivalence depends, in principle, on $G$ and is weaker than elementary equivalence. For every connected non-solvable compact (hence non Abelian) Lie group $G$, the holonomy equivalence agrees with elementary equivalence in the analytic and in the smooth immersive case [22, 26]. In the smooth non-immersive case this is no longer 
true: a simple example of a smooth loop giving a non trivial elementary equivalence class, but with trivial holonomies for any $G$, has been given in [17].

For $G=T^{n}$, a torus in dimension $n, \quad n \geq 1$, the two equivalence relations are different. However, the holonomy equivalence does not depend on $n$. In the Abelian case, the group $\operatorname{Loop}_{\star}(M)$ quotients to a group, we will denote by $\operatorname{Hoop}_{\star}(M)$, since holonomy equivalence classes of loops were first introduced in [6] and called hoops. Holonomy equivalence classes of paths in $\operatorname{Path}(M)$ form a groupoid, which we analogously denote by $\operatorname{Hath}(M)$.

The group of gauge transformations is the group $G a u=C^{\infty}(M, G)$, acting on $\mathcal{A}$ by $A . g=g^{-1} A g+g^{-1} d g$ where $d$ denotes the exterior derivative. The corresponding action on parallel transports is given by

$$
H_{A . g}(\gamma)=g(\gamma(1))^{-1} H_{A}(\gamma) g(\gamma(0)) \quad \gamma \in \operatorname{Path}(M)
$$

and is the restriction to Gau of the action of $G^{M}$. Of course, $\mathcal{A}$ is not $G^{M}$-invariant, however its $G a u$-invariance implies that $\mathcal{A}_{\star}$ is $A d G$-invariant. Since $G a u$ is dense in $G^{M}$ and the action of $G^{M}$ on $\operatorname{Hom}(\operatorname{Path}(M), G)$ is continuous, the Gau-invariance of $\mathcal{A}$ implies the $G^{M}$-invariance of $\overline{\mathcal{A}}$. We recall that every function $f$ in $\operatorname{Cyl}(\mathcal{A})$ can be uniquely extended to a continuous function on $\overline{\mathcal{A}}$, which is $G^{M}$-invariant if $f$ is Gau-invariant. As a consequence, $\operatorname{Cyl}_{\text {Gau }}(\mathcal{A})=$

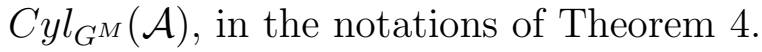

The projection $\mathcal{P}_{\star}: \operatorname{Hom}(\operatorname{Path}(M), G) \rightarrow \operatorname{Hom}\left(\operatorname{Loop}_{\star}(M), G\right)$ quotients to a bijection

$$
\mathcal{A} / \text { Gau } \leftrightarrow \mathcal{A}_{\star} / A d G
$$

(see [3]). Analogous statements can be done using $\operatorname{Path}(M)$ and $\operatorname{Loop}_{\star}(M)$ and also for the weaker differentiability conditions on paths.

In the non-perturbative quantization program a standing point is to give a compactification of the configuration space $\mathcal{A} / G a u$, i.e. to embed the configuration space in the spectrum of some $A d G$-invariant cylindrical algebra on $\mathcal{A}_{\star}$. A compactification of $\mathcal{A}$ can be achieved by the embedding in the spectrum of the cylindrical algebra of $\mathcal{A}$. One is interested in studying the consistence of these compactifications.

The analytic case.

In the analytic case $\mathcal{A}$ is viewed as a subset of $\operatorname{Hom}(\operatorname{Path}(M), G)$. To distinguish this case from the smooth one, we will denote by $\operatorname{Cyl}(\mathcal{A})$ the analytic cylindrical algebra and by $\operatorname{Hol}\left(\mathcal{A}_{\star}\right)$ the analytic holonomy algebra, i.e. the Wilson $\mathbb{C}^{*}$-algebra of $\mathcal{A}_{\star}$.

In this setting the following strong version of the Approximation Condition on $\mathcal{A}$ is assured: 
For every finite set of paths $\left\{\gamma_{k}\right\}_{k=1, \ldots, r}$ and every homomorphism $H: \operatorname{Path}(M) \rightarrow G$ there exists a smooth connection $A$ on $M \times G$ such that

$$
H\left(\gamma_{k}\right)=H_{A}\left(\gamma_{k}\right) \quad k=1, \ldots, r
$$

The property has been proved in [6] for $\gamma_{k} \in \operatorname{Loop}_{\star}(M)$ ( see also [26]) and the proof extends easily to $\gamma_{k} \in \operatorname{Path}(M)$. Owing to Theorem 1 we can conclude that

$$
\operatorname{Spec}(\operatorname{Cyl}(\mathcal{A})) \equiv \overline{\mathcal{A}}=\operatorname{Hom}(\operatorname{Path}(M), G) .
$$

As a consequence, we obtain that

$$
\operatorname{Spec}\left(\mathrm{Cyl}_{G a u}(\mathcal{A})\right) \equiv \operatorname{Hom}\left(\operatorname{Loop}_{\star}(M), G\right) / A d G \equiv \overline{\mathcal{A} / G a u} .
$$

In the special case that $G$ is a closed normal subgroup of $U_{\mathrm{n}}$, the unitary equivalence classes of $G$-valued homomorphisms agree with their conjugation classes, so that $\mathcal{A} / G a u \equiv \widehat{\mathcal{A}_{\star}}=\mathcal{A}_{\star} / A d G$. By Theorem 5 we get

$$
\operatorname{Spec}\left(\operatorname{Hol}\left(\mathcal{A}_{\star}\right)\right) \equiv \widehat{\operatorname{Hom}}\left(\operatorname{Loop}_{\star}(M), G\right)=\overline{\mathcal{A} / G a u} .
$$

Dually, we have that

$$
\operatorname{Hol}\left(\mathcal{A}_{\star}\right)=\mathrm{Cyl}_{A d G}\left(\mathcal{A}_{\star}\right) \equiv \mathrm{Cyl}_{\text {Gau }}(\mathcal{A}) .
$$

However, the analytic case is not really satisfactory from the physical point of view, since for applications to the loop quantum gravity one needs to consider diffeomorphism invariance on the closure of $\mathcal{A} / G a u$ while Loop $_{\star}(M)$ is invariant only w.r.t. analytic diffeomorphisms.

The Abelian smooth immersive case.

Ashtekar and Lewandowski studied in [6] the case of $G=U(1)$, working in the piecewise $C^{1}$ setting. They denoted by $\mathcal{H} \mathcal{G}$ the group of hoops obtained by the holonomy equivalence classes of piecewise $C^{1}$ loops based on $\star$ and considered in the compact space $\operatorname{Hom}(\mathcal{H G}, U(1))$ the subset $\mathcal{A}_{\star}$ obtained by the holonomy maps of the smooth connections. They proved that

$$
\overline{\mathcal{A}_{\star}}=\operatorname{Hom}(\mathcal{H G}, U(1)) .
$$

Their proof works also in the case of a general torus $T^{n}$ in any dimension $n$ and for every differentiability class of loops and hoops. It works also in the smooth immersive category, when the group $\mathcal{H} \mathcal{G}$ is replaced by the group $\mathrm{Hoop}_{\star}(M)$, so that

$$
\overline{\mathcal{A}_{\star}}=\operatorname{Hom}\left(\operatorname{Hoop}_{\star}(M), T^{n}\right) .
$$

Let us consider the smooth connections $\mathcal{A}$ as a subset of $\operatorname{Hom}\left(\operatorname{Hath}(M), T^{n}\right)$. The gauge invariance of $\mathcal{A}$ implies the $G^{M}$-invariance of $\overline{\mathcal{A}}$. Moreover, 
$\mathcal{A}_{\star}$ is $A d G$-invariant. By the last statement in Theorem 4 we obtain that

$$
\overline{\mathcal{A}}=\operatorname{Hom}\left(\operatorname{Hath}(M), T^{n}\right)
$$

and by $\mathcal{A} /$ Gau $\equiv \mathcal{A}_{\star}$ we have

$$
\overline{\mathcal{A} / \mathrm{Gau}} \equiv \operatorname{Hom}\left(\operatorname{Hoop}_{\star}(M), T^{n}\right) .
$$

The above quoted result allows one to characterize also the closure of $\mathcal{A}$ in the space $\operatorname{Hom}\left(\operatorname{Path}(M), T^{n}\right)$ : we can identify $\operatorname{Hom}\left(\operatorname{Hath}(M), T^{n}\right)$ with the closed subgroup of $\operatorname{Hom}\left(\operatorname{Path}(M), T^{n}\right)$, consisting of the homomorphisms which are constant on each holonomy equivalence class of paths, obtaining

$$
\overline{\mathcal{A}}=\operatorname{Hom}\left(\operatorname{Hath}(M), T^{n}\right) \subset \operatorname{Hom}\left(\operatorname{Path}(M), T^{n}\right) .
$$

Fleischhack proves in [16] that $\overline{\mathcal{A}}$ is a proper subset in $\operatorname{Hom}\left(\operatorname{Path}(M), T^{n}\right)$. Analogously, one recognizes that $\left.\operatorname{Hom}_{\mathrm{oroop}}(M), T^{n}\right)$ is a closed proper subgroup of $\operatorname{Hom}\left(\operatorname{Loop}_{\star}(M), T^{n}\right)$ and agrees with the closure of $\mathcal{A}_{\star}$ in $\operatorname{Hom}\left(\operatorname{Loop}_{\star}(M), T^{n}\right)$.

In contrast, in the analytic case it is well known 26 that the group Hoop $_{\star}(M)$ is the quotient of Loop $(M)$ by its commutator group, so that $\operatorname{Hom}\left(\operatorname{Loop}_{\star}(M), T^{n}\right) \equiv \operatorname{Hom}\left(\operatorname{Hoop}_{\star}(M), T^{n}\right)$ and, analogously, $\operatorname{Hom}\left(\operatorname{Path}(M), T^{n}\right) \equiv \operatorname{Hom}\left(\operatorname{Hath}(M), T^{n}\right)$.

The general smooth immersive case.

Our aim is to characterize the closure of $\mathcal{A}$ in the smooth immersive case for a connected compact Lie group $G$. Fleischhack has proved that $\mathcal{A}$ is dense in $\operatorname{Hom}(\operatorname{Path}(M), G)$ only for connected and semisimple $G$ [16. The same author will discuss denseness of connections in the nonimmersive case and for more general categories of immersive paths in [19.

The space $\overline{\mathcal{A}}$ was investigated in the smooth immersive case for any connected compact Lie group $G$ by Baez and Sawin in 10. They associate to every finite ordered family $C=\left(c_{1}, \ldots, c_{r}\right)$ of piecewise immersed or constant paths the map $p_{C}: \mathcal{A} \rightarrow G^{r}, H_{C}(A)=\left(H_{c_{1}}(A), \ldots, H_{c_{r}}(A)\right)$ and studied its range $\mathcal{A}_{C}$. They found special families of independent paths, the webs, for which this range can be characterized. We recall that a family $C$ of paths is said to be independent if a path $\lambda$ in $C$ cannot be decomposed using other paths in $C$ or their inverses. A family $C$ depends on another family $C^{\prime}$ if every path in $C$ can be obtained from paths in $C^{\prime}$ or their inverses by using the path composition.

The definition of a web is quite involved and we refer to the quoted authors. Their main result is that every finite family $C$ of paths depends on a web. Moreover it was proved in [22] (see also [26]) that if 
$G$ is semisimple, for every web $W$ the range $\mathcal{A}_{W}$ is exactly $G^{r}$, where $r$ is the cardinality of $W$.

Joining the above results one obtains that

$$
\overline{\mathcal{A}}=\operatorname{Hom}(\operatorname{Path}(M), G)
$$

for every connected compact semisimple Lie group $G$.

Let now $G$ be isomorphic to the product of a torus $T^{n}$ and a connected compact semisimple Lie group $S$. Then one obtains that

$$
\operatorname{Hom}(\operatorname{Path}(M), G)=\operatorname{Hom}\left(\operatorname{Path}(M), T^{n}\right) \times \operatorname{Hom}(\operatorname{Path}(M), S)
$$

and that

$\operatorname{Hom}\left(\operatorname{Loop}_{\star}(M), G\right) / A d G=\operatorname{Hom}\left(\operatorname{Loop}_{\star}(M), T^{n}\right) \times \operatorname{Hom}\left(\operatorname{Loop}_{\star}(M), S\right) / A d S$.

The Lie algebra $\mathfrak{g}$ splits as $\mathfrak{g}=\mathbf{R}^{n}+\mathfrak{s}$, so that a connection $A$ on $M \times G$ can be identified with a couple $A_{T^{n}}$ and $A_{S}$ of 1 -forms on $M$ taking values in the Lie algebras $\mathbf{R}^{n}$ and $\mathfrak{s}$ of $T^{n}$ and $S$, respectively. It follows from the definition of path ordered integral that $H_{A}(\lambda)=$ $H_{A_{T^{n}}}(\lambda) H_{A_{S}}(\lambda)$ for every $\lambda \in \operatorname{Path}(M)$.

Theorem 6. Let $G=T^{n} \times S$ the product of a torus $T^{n}$ and a connected compact semisimple Lie group $S$. The closure of the set $\mathcal{A}$ in $\operatorname{Hom}(\operatorname{Path}(M), G)$ is $\operatorname{Hom}\left(\operatorname{Hath}(M), T^{n}\right) \times \operatorname{Hom}(\operatorname{Path}(M), S)$. The closure of $\mathcal{A} /$ Gau in Hom $\left(\operatorname{Loop}_{\star}(M), G\right) / A d G$ is Hom $\left(\operatorname{Hoop}_{\star}(M), T^{n}\right) \times$ $\operatorname{Hom}\left(\operatorname{Loop}_{\star}(M), S\right) / A d S$.

Proof. This is an immediate consequence of the above remarks and of the results in the Abelian and the semisimple cases, respectively.

Let us recall that every compact connected Lie group $G$ is of the form $\left(T_{0} \times S\right) / K$ where $T_{0}$ is the identity component of the centre of $G, S$ is a connected compact semisimple Lie group and $K$ is a finite group contained in the centre of $T_{0} \times S$. By the general theory of compact Abelian Lie groups, $T_{0}$ is trivial or it is a torus. We denote by $p_{K}$ the projection $T_{0} \times S \rightarrow G$ and by $\left(p_{K}\right)_{*}: \operatorname{Hom}\left(\operatorname{Path}(M), T_{0} \times S\right) \rightarrow$ $\operatorname{Hom}(\operatorname{Path}(M), G)$ the map defined by $\left(p_{K}\right)_{*}(H)=p_{K} \circ H$.

The case where $T_{0}$ is a torus is discussed in the following theorem.

Theorem 7. Let $G$ be a compact connected Lie group represented in the form $G=\left(T^{n} \times S\right) / K$, as above. The closure of $\mathcal{A}$ in $\operatorname{Hom}(\operatorname{Path}(M), G)$ is $\left(p_{K}\right)_{*}\left(\operatorname{Hom}\left(\operatorname{Hath}(M), T^{n}\right) \times \operatorname{Hom}(\operatorname{Path}(M), S)\right)$. The closure of $\mathcal{A} / G a u$ in $\operatorname{Hom}\left(\operatorname{Loop}_{\star}(M), G\right) / A d G$ is $\left(p_{K}\right)_{*}\left(\operatorname{Hom}_{(H o o p}(M), T^{n}\right) \times$ $\left.\left.\operatorname{Hom}\left(\operatorname{Loop}_{\star}(M), S\right)\right) / A d S\right)$. 
Proof. The commutative diagram holds:

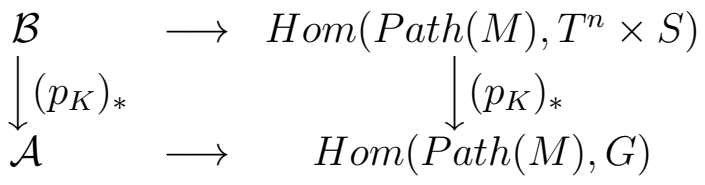

where $\mathcal{B}$ denotes the space of holonomies of smooth connections on $M \times\left(T^{n} \times S\right)$. Since the Lie algebras $\mathbf{R}^{n}+\mathfrak{s}$ and $\mathfrak{g}$ are isomorphic, the bundles $M \times\left(T^{n} \times S\right)$ and $M \times G$ have isomorphic connection one-forms, hence $\left(p_{K}\right)_{*}(\mathcal{B})=\mathcal{A}$.

The map $\left(p_{K}\right)_{*}$ is continuous in the Tychonoff topologies and is closed, since $\operatorname{Hom}\left(\operatorname{Path}(M), T^{n} \times S\right)$ is compact. Therefore $\left(p_{K}\right)_{*}(\overline{\mathcal{B}})=$ $\overline{\left(p_{K}\right)_{*}(\mathcal{B})}=\overline{\mathcal{A}}$. Then we use Theorem 6 .

To prove the second statement, we have to apply analogous arguments to the diagram

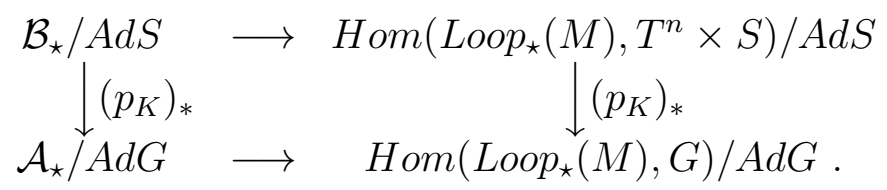

The only non trivial point to prove is that the (quotiented) projection $\left(p_{K}\right)_{*}: \mathcal{B}_{\star} / A d S \rightarrow \mathcal{A}_{\star} / A d G$ is onto. This follows immediately by $\left(p_{K}\right)_{*}\left(\mathcal{B}_{\star}\right)=\mathcal{A}_{\star}$.

We can characterize the spectra of the smooth immersive cylindrical algebras $C y l(\mathcal{A})$ and $C y l_{\text {Gau }}(\mathcal{A})$ and of the smooth immersive holonomy algebra $\operatorname{Hol}\left(\mathcal{A}_{\star}\right)$.

For a semisimple connected compact Lie group $G$ we have

$$
\operatorname{Spec}(\operatorname{Cyl}(\mathcal{A})) \equiv \overline{\mathcal{A}}=\operatorname{Hom}(\operatorname{Path}(M), G)
$$

and

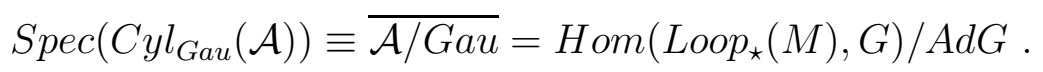

In the special case that $G$ is a normal subgroup of $U(\mathrm{n})$, e.g. if $G$ is $S U(\mathrm{n}), \mathrm{n}>1$, we identify $\operatorname{Hol}\left(\mathcal{A}_{\star}\right)$ with $C y l_{\text {Gau }}(\mathcal{A})$ and we have

$$
\operatorname{Spec}\left(\operatorname{Hol}\left(\mathcal{A}_{\star}\right)\right) \equiv \overline{\mathcal{A} / \operatorname{Gau}}=\widehat{\operatorname{Hom}}\left(\operatorname{Loop}_{\star}(M), G\right) .
$$

For $G=T^{n} \times S$, with semisimple $S$, we have

$$
\operatorname{Spec}(C y l(\mathcal{A}))=\operatorname{Hom}\left(\operatorname{Hath}(M), T^{n}\right) \times \operatorname{Hom}(\operatorname{Path}(M), S)
$$

and

$\operatorname{Spec}\left(C y l_{G a u}(\mathcal{A})\right)=\operatorname{Hom}\left(\operatorname{Hoop}_{\star}(M), T^{n}\right) \times \operatorname{Hom}\left(\operatorname{Loop}_{\star}(M), S\right) / \operatorname{Ad} S$. 
Finally, for $G=\left(T^{n} \times S\right) / K$, as above, we have

$$
\operatorname{Spec}(\operatorname{Cyl}(\mathcal{A}))=\left(p_{K}\right)_{*}\left(\operatorname{Hom}\left(\operatorname{Hath}(M), T^{n}\right) \times \operatorname{Hom}(\operatorname{Path}(M), S)\right)
$$

and

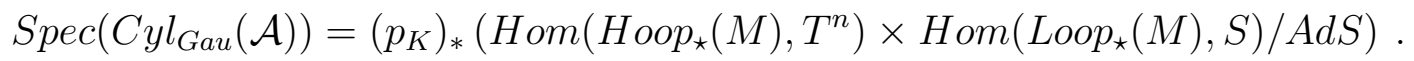

\section{NON TRIVIAL BUNDLES}

We will extend the above results to the general case that $\mathcal{A}$ is the set of smooth connections of a non trivial principal bundle $P(M, G)$.

For $x, y \in M$ we denote by $E q\left(P_{x}, P_{y}\right)$ the space of the $G$-equivariant maps from the fiber $P_{x}$ to the fiber $P_{y}$. This space consists of invertible maps and every choice of $u_{x} \in P_{x}$ and $u_{y} \in P_{y}$ defines a bijection of $E q\left(P_{x}, P_{y}\right)$ onto $G$, so $E q\left(P_{x}, P_{y}\right)$ becomes a compact space whose topology does not depend on the choice. The disjoint union $E q(P)=$ $\coprod_{x, y \in M} E q\left(P_{x}, P_{y}\right)$ is a groupoid, since $\phi_{y, x} \in E q\left(P_{x}, P_{y}\right)$ and $\phi_{y^{\prime}, x^{\prime}} \in$ $E q\left(P_{x^{\prime}}, P_{y^{\prime}}\right)$ can be composed if $y=x^{\prime}$.

Let we denote by $\Lambda$ any of the groupoids of paths previously introduced and consider the space $\operatorname{Hom}(\Lambda, E q(P))$ of groupoid homomorphisms of $\Lambda$ in $E q(P)$. The space $\operatorname{Hom}(\Lambda, E q(P))$ is compact as a closed subset of the product $\prod_{\lambda \in \Lambda} E q\left(P_{s(\lambda)}, P_{r(\lambda)}\right)$, which is compact in the Tychonoff topology.

We fix a point $\star$ in $M$ and introduce the compact space $\mathcal{E}_{\star}$ of the maps $\eta \in \prod_{x \in M} E q\left(P_{\star}, P_{x}\right)$ satisfying $\eta(\star)=i d$. For every $x \in M$, we fix a path $e_{x} \in \Lambda$ with $s\left(e_{x}\right)=\star$ and $r\left(e_{x}\right)=x$, choosing $e_{\star}=\star$. To every $H \in \operatorname{Hom}(\Lambda, E q(P))$ we associate the element $\eta_{H} \in \mathcal{E}_{\star}$ given by $\eta_{H}(x)=H\left(e_{x}\right)$.

Fixed a point $u_{\star}$ in $P_{\star}$, we identify the group $E q\left(P_{\star}, P_{\star}\right)$ with the group $G$ and define the map $\mathcal{P}_{\star}: \operatorname{Hom}(\Lambda, E q(P)) \rightarrow \operatorname{Hom}\left(\Lambda_{\star}, G\right)$ which associates to each $H \in \operatorname{Hom}(\Lambda, E q(P))$ its restriction to $\Lambda_{\star}$.

As in section 2, the map $H \mapsto\left(\mathcal{P}_{\star}(H), \eta_{H}\right)$ is a homeomorphism

$$
\Theta: \operatorname{Hom}(\Lambda, E q(P)) \rightarrow \operatorname{Hom}\left(\Lambda_{\star}, G\right) \times \mathcal{E}_{\star} .
$$

Let us recall that $\operatorname{Gau}(P)$ is the group of the smooth sections of the fiber bundle $\coprod_{x \in M} E q\left(P_{x}, P_{x}\right) \rightarrow M$, with fiber $G$. Gau $(P)$ is dense in the group $\overline{\operatorname{Gau}(P)}$ of all sections of this bundle, a compact group isomorphic to $G^{M}$ (see [0]).

Actions of $\overline{\operatorname{Gau}(P)}$ on $\operatorname{Hom}(\Lambda, E q(P))$, on $\operatorname{Hom}\left(\Lambda_{\star}, G\right)$ and on $\mathcal{E}_{\star}$ are defined as follows:

$$
\begin{array}{ll}
(H \phi)(\lambda)=\phi(r(\lambda))^{-1} H(\lambda) \phi(s(\lambda)) & H \in \operatorname{Hom}(\Lambda, E q(P)), \\
\left(H_{\star} \phi\right)(\gamma)=A d_{\phi(\star)-1} H_{\star}(\gamma) & H_{\star} \in \operatorname{Hom}\left(\Lambda_{\star}, G\right), \\
(\eta \phi)(x)=\phi^{-1}(x) \eta(x) \phi(\star) & \eta \in \mathcal{E}_{\star}
\end{array}
$$


for every $\phi \in \overline{\operatorname{Gau}(P)}$.

The map $\Theta$ is equivariant and quotients to a homeomorphism of $\operatorname{Hom}(\Lambda, E q(P)) \overline{\operatorname{Gau}(P)}$ onto $\operatorname{Hom}\left(\Lambda_{\star}, G\right) / A d G$.

Cylindrical functions and algebras can be treated for the space $\mathcal{A}$ of connections on a general bundle. A cylindrical function is a function of the form $F_{\lambda_{1}, \ldots, \lambda_{r} ; f}(A)=f\left(H_{A}\left(\lambda_{1}\right), \ldots, H_{A}\left(\lambda_{r}\right)\right)$, where $f$ is a continuous function on the compact space $E q\left(P_{s\left(\lambda_{1}\right)}, P_{r\left(\lambda_{1}\right)}\right) \times \ldots \times E q\left(P_{s\left(\lambda_{r}\right)}, P_{r\left(\lambda_{r}\right)}\right)$.

Analogous embedding results to the ones given in section 2 and section 3 can be easily worked out for the spectrum of these cylindrical algebras. Analogously to the trivial case, we obtain

$$
\operatorname{Spec}(\operatorname{Cyl}(\mathcal{A})) \equiv \overline{\mathcal{A}} \subset \operatorname{Hom}(\Lambda, E q(P)),
$$

and

$$
\operatorname{Spec}\left(\operatorname{Cyl}_{\operatorname{Gau}(P)}(\mathcal{A})\right) \equiv \overline{\mathcal{A} / \operatorname{Gau}(P)} \subset \operatorname{Hom}\left(\Lambda_{\star}, E q(P)\right) / A d G .
$$

For the groups $U(\mathrm{n})$ or $S U(\mathrm{n})$ we have that

$$
\operatorname{Hol}\left(\mathcal{A}_{\star}\right)=\operatorname{Cyl}_{\operatorname{Gau}(P)}(\mathcal{A})
$$

and that

$$
\operatorname{Spec}\left(\operatorname{Hol}\left(\mathcal{A}_{\star}\right)\right) \equiv \overline{\mathcal{A} / \operatorname{Gau}(P)} \subset \operatorname{Hom}\left(\Lambda_{\star}, E q(P)\right) / A d G .
$$

However, the concrete characterization of the closure of $\mathcal{A}$ in the non trivial case remains an open problem. In the analytic case, the Approximation Condition on $\mathcal{A}$ is proved for any $G$ (see e.g. [26]). In the smooth immersive case, the Approximation Condition can be proved for a semisimple group $G$ using the fact that webs are decomposed in tassels, each contained in some trivializing neighborhood, as in the proof of Proposition 2 in 10 .

The results obtained in the Abelian non-analytic case are difficult to be generalized to non trivial bundles, since hoops are not local. Ashtekar and Lewandowski proved that $\overline{\mathcal{A}_{\star}}=\operatorname{Hom}(\mathcal{H} \mathcal{G}, U(1))$ for the Hopf bundle and its pullbacks [6].

Acknowledgements. The authors would like to thank C. Fleischhack for his comments on the manuscript and for signaling out his results contained in [16], [17] and [19].

\section{REFERENCES}

[1] M. C. Abbati and A. Manià, On differential structure for projective limits of manifolds, J. Geom. Phys. 29 (1999), 35-63.

[2] M.C. Abbati, A. Manià and E. Provenzi, Inductive Construction of the Loop Transform for Abelian Gauge Theories, Lett. Math. Phys. 57 (2001), 69-81. 
[3] J. Anandan, Quantum Interference and the Classical Limit, Int. J. Theor. Phys. 19 N. 7 (1980), 537-556.

[4] A. Ashtekar and C. Isham, Representations of the holonomy algebras of gravity and non-Abelian gauge theories, Class. Quant. Grav. 9 (1992), 1433-1485.

[5] A. Ashtekar, J. Lewandowski, D. Marolf, J.Mourão and T. Thiemann, Quantization of diffeomorphism invariant theories of connections with local degrees of freedom, J. Math. Phys. 3611 (1995), 6456-6493.

[6] A. Ashtekar and J. Lewandowski, Representation Theory of analytic holonomy $\mathbb{C}^{*}$ algebras, in: J. C. Baez (ed.), Knots and Quantum Gravity, Oxford Univ. Press. (1994) pp. 21-61.

[7] A. Ashtekar and J. Lewandowski, Projective techniques and functional integration for gauge theories, J. Math. Phys. 36 (1995), 2170-2191.

[8] J. C. Baez, Diffeomorphism-invariant Generalized Measures on the Space of Connections Modulo Gauge Trasformations, in L. Crane and D. Yetter (eds.), The Proceedings of the Conference on Quantum Topology, World Scientific (1994), pp. 213-223.

[9] J. C. Baez, Spin Networks in Nonperturbative Quantum Gravity, in L. Kaufmann (ed.), The interface of Knots and Physics, A.M.S. (1996), pp. 167-203.

[10] J. C. Baez and S. Sawin, Functional Integration on spaces of Connections, J. Funct. Analysis 150 (1997), 1-26.

[11] J. C. Baez and S. Sawin, Diffeomorphism-Invariant Spin Network States, J. Funct. Analysis 158 (1998) 253-266.

[12] H. Baumgärtel, On a theorem of Ashtekar and Lewandowski in the mathematical framework of canonical quantization of quantum gravity, in: H. J. Schmidt and M. Rainer (eds), Current Topics in Mathematical Cosmology, Proc. Int. Seminar, World Scientific (1998), pp. 217-222.

[13] T.Bröcker, T.tom Dieck, Representations of Compact Lie Groups, G.T.M. 98 Springer (1995).

[14] J. Dixmier, Les $\mathbb{C}^{*}$-algèbres et leurs représentations, Gautier-Villars (1969).

[15] S. Eilenberg and N Steenrod, Foundations of Algebraic Topology, Princeton University Press (1952).

[16] C. Fleischhack, Mathematische und physikalische Aspekte verallgemeinerter Eichfeldtheorien im Ashtekarprogramm, PhD thesis, Universität Leipzig (2001).

[17] C. Fleischhack, Hyphs and the Ashtekar-Lewandowski Measure, to appear in J. Geom. and Phys. arXiv:math-ph/0001007.

[18] C. Fleischhack, Stratification of the Generalized Gauge Orbit Space, Commun. Math. Phys. 214 (2000) 607-649.

[19] C. Fleischhack, Regular Connections among Generalized Connections, in preparation.

[20] R. Giles, Reconstruction of gauge potentials from Wilson loops, Phys. Rew. D 24 N. 8 (1981), 2160-2168.

[21] S. Kobayashi and K. Nomizu, Foundations of Differential Geometry, Vol I, Wiley (1963).

[22] J. Lewandowski and T. Thiemann, Diffeomorphism invariant Quantum Field Theories of Connections in terms of webs, Class. Quant. Grav. 16 (1999), 2299-2322.

[23] D. Marolf and J. M. Mourão, On the Support of the Ashtekar-Lewandowski Measure, Comm. Math. Phys. 170 (1995), 583-605.

[24] J. M. Mourão, T. Thiemann and J. M. Velhinho, Physical Properties of Quantum Field Theory Measures, J. Math. Phys. 40 (1999) 2337-2353.

[25] C. Rovelli and L. Smolin, Loop Space Representation of Quantum General Relativity, Nuclear Physics B331 (1990), 80-152.

[26] P. Spallanzani, Groups of loops and hoops, Comm. Math. Phys. 216 (2001), 243-253.

[27] J. M. Velhinho, A groupoid approach to spaces of generalized connections, arXiv:hept-th/0011200. 